\title{
A TREATMENT OF FUNCTIONAL DEFINITE DESCRIPTIONS
}

\author{
Hajime Wada \\ Intelligent Text Processing, Inc. \\ 1310 Montana, Suite 201, Santa Monica, CA 90403, USA \\ hw@itpinc.com
}

\section{INTRODUCTION}

Functional anaphoric expressions are referring expressions whose references are identified with respect to references of other objects in a discourse. Among a few types of functional anaphoric expressions such as Wh expressions and pronouns (Cooper 1979, Engdahl 1984, Kamp 1984, Chierchia 1993), definite descriptions provide a locution for functional expressions. A typical example is as follows:

(1) Every book about Picasso made the anthor rich.

In (1), 'the author' does not have its usual sense of the antecedent, such as 'an author', anywhere in the discourse. Instead, the reference of 'the author' is detcrmined with respect to the reference of 'book'. In other words, the description, 'author', works as a function that takcs a referent for 'book' as an argument and returns a value that is the referent for 'author'. Kamp (1984) calls this kind of expressions Functional Definite Descriptions (hence, we follow him here, and call them FDD for short, and DD for definite descriptions). In this paper, I will call 'book' a functional antecedent of FDD 'author' and its resulting anaphoric link between 'book' and 'author' a functional anaphoric link.

This paper describes a classification of Functional Definite Descriptions and proposes an analysis of FDD based on a claim that FDD behave more like pronominals than definite descriptions. This paper also reports an implementation of the treatment described here in an English text understanding system, Interpretext, at ITP.

\section{RANGE OF FDD}

When we process real texts, it is apparent that FDD is a non-trivial part of the use of definite descriptions. FDD constitutes a large portion of DD use among so-called firstmention use. In a recent study of a Swedish corpus, Fraurud (1990) reports that $60.9 \%$ of total DD occurrences are firstmention use, and such complex DI) as the ones in the form of 'the $X$ of $Y$ ' in corresponding English structures (a typical form of FDD) accounts for $41.2 \%$ among the first-mention usc. Since FDD can be found among simple DD, the percentage of FDD further increases. Although Fraurud's study reveals its importance in Swedish, it is easy to assume a comparable situation in English. In literature, FDD is usually regarded as a limited phenomenon that is difficult to formalize. However, such a view toward FDD is shortsighted since it not only undermines the importance of IDD with respect to other use of DD, but also misses the important relations to functional anaphoric expressions in other cat- egories.

Hawkins (1978) studies various usage of definite descriptions and proposes an analysis based on the theory of Familiarity. Two of his usage of the first-mention definites are FIDD under consideration here: an associative use such as 'a car' - 'the steering wheel', and a larger situation use such as 'a town' - 'the church'. IIe claims that in these cases common knowledge shared by a speaker and the hearer is very general and inferable from lexical information without pragmatic information. Hawkins' study signifies the importance of Familiarity presupposition of definite descriptions as well as the range of definite descriptions although his study covers only the major usage and misses some important issues, which we will examine below.

Kamp (1984) provides the first but very insightful formal semantic analysis of FDD. In Kamp's Discourse Representation theoretic treatment of FDD, a head noun of FDD always introduces a new reference marker for the individual that it denotes and a functional anaphoric referent into a universe of the DRS. He notes that FDD ranges over types of functional referents. That is, if the functional referent of FDD is pronominal, the FDD needs to utilize pronominal resolution mechanism, and if demonstrative, then demonstrative resolution mechanism. Kamp's work suggests the range of FDD distribution is wider than that suggested by Hawkins and shows us a point of departure.

I obner(1985) proposes an analysis of DD based on a lexical distinction among nouns; sortal nouns, functional nouns, and non-functional relational nouns. Sortal nouns are typical nouns that denotes individuals. Functional nouns are relational nouns with situational arguments. Ile studies a wide range of FDDs and classifies many of them into a bag of functional nouns. $\Lambda$ s a result, functional nouns include 'weather', 'time', 'sun', 'speaker', 'hearer', 'president', 'referee', 'bride', 'head', 'top', 'surface', 'hcight', 'weight', 'birth', 'death', 'beginning', 'end', etc. He points out that functional nouns allow modifications with pp, adjectives and adverbs, but non-functional nouns do not. Thus, the fact that 'the present wife' is acceptable but 'the present son' is not indicates the difference between functional nouns and nonfunctional relational nouns. However, even seemingly noncontroversial relational noun such as 'daughter' can find certain situation where it is modified by a pp. For example, a man has two daughters, one studying in L.A. and the other working in Seattle. We may refer to his daughters 'the daughter in L.A. and the daughter in Seattle'. This difficulty 
in distinguishing functional and non-functional nouns is one of the major problems in Lobner's treatment. Another major problem is the lack of constraining mechanism on linking. For example,

(2)

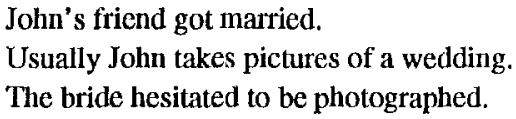

It is not difficult for normal English speakers to identify the function of the second sentence as a background information. 'Therefore, the intended functional link from 'the bride' in the third sentence should be to the first sentence. However, Lobner's analysis incorrectly allows a link between 'the bride' in the third sentence and 'a wedding' in the second sentence.

In a recent study, Chierchia (1993) proposes a treatment of FDD based on his theory of Dynamic Binding. Chierchia represents functional anaphoric links by co-indexing a functional antecedent with a superscript and I'DD with a subscript as shown below. IIe calls this a-indexation.

(1') Every book ${ }^{\mathbf{i}}$ about Picasso made the author ${ }_{i}$ rich.

IIe claims a-indexed anaphoric links are comparable to pronominal anaphoric links. This means that FDD should follow the same constraints on the pronominal anaphoric links. The idea behind this claim is very similar to Kamp's idea for elliptic FDD. That is, both studies note certain similarity between FDD and pronominal possessives. In effect, both studies can explain why the following FDD link is not felicitous in terms of accessibility of pronominal anaphora.

(3) Lvery book ${ }^{i}$ about Picasso was published by Mr.King.

\#The author ${ }_{\mathbf{i}}$ became rich.

However, Chierchia's study does not consider other FDD, those that Kamp analyzes. Also, it fails to explain a pair of sentences such as follows:

(4) a. Usually if John meets every pastor who administers a wedding $i$, he writes to the bride $i$. b. \#Usually if John mcets every boy who has an interesting book ${ }^{i}$, he writes to the author ${ }_{i}$.

Notice that (4-a) and (4-b) are structurally identical while FDD is felicitous in (4-a) but infelicitous in (4-b).

In what follows, I will present a classification of FDDs and their analyses based on DRT.

\section{TWO CLASSES OF FDD}

In this paper, I propose that FDD should be classified into two basic types according to their semantic characteristics. The first class is called relational FDD and the second non-relational FDD. Relational FDD denotes relations between objects and these relations are lexical properties of head nouns of FDD. Kinship terms such as 'son', 'mother', 'sister', etc. are typical examples of relational nouns. Nonrelational FDD denote functions from sets of individuals to individuals. A typical example is a superlative noun phrase such as 'the tallest tree'. Ordinal number modification such as 'the third man' and identifying adjective modification such as 'the identical book' are other possible examples of this type. The distinction between relational and nonrelational FDD is characterized below:

1-i) relational FDD takes an object in a given discourse as its functional antecedent and forms a functional anaphoric link,

1-ii) such a functional anaphoric link is licensed by lexical characteristics of the functional description of the head nouns,

and 1-iii) relational FDD and their functional antecedents can form a construction of "FDD of (antecedent)",

while

2-i) non-relational YDD takes a sclection set instead of an object as a functional antecedent, and 2-ii) the link is licensed by a modifier such as superlative, ordinal number, or identifying adjective rather than a head noun of FDD.

\section{RELATIONAL FDD}

Functional anaphoricity of Relational FDD is marked by a head noun's lexical property. That is, only relational nouns can be heads of relational FDD. There are certain subclasses of relational FDDs. I propose three subclasses: FDD based on i) lexical relations, ii) temporal/locational and iii) situational roles. Lexical relational FDD is characterized by their paraphrasability with true possessives (that is, both forms of "X's Y" and "Y of X"). In effect, this subclass constitutes the largest group among FDDs. Situational Role FIDD is marked by social roles and professions such as 'judge' and 'bride'. Another subclass, temporal/ locational FDD is marked by relations with time and location.

\subsection{FDD based on Lexical Relations \\ Possessives and Relations}

This class is marked by the paraphrasability to possessive constructions. That is, I assume that FDD with lexical relations must be paraphrased by both forms of " $X$ 's $Y$ " and "the $Y$ of $X$ ". In other words, if English lexicon includes concepts such as relations denoted by certain class of nouns, this information must be shared by many constructions in English, and I assume that it is the case that possessives, compound nouns, and FDD utilize this information. Furthermore, possessive paraphrasability of this type of FDD captures their similarity to the pronominal anaphor that appear in possessive constructions as possessor pronominals. If this is correct, then it predicts that this type of FDD obeys the constraints on anaphoric links that pronominals obey.

Barker (1991) proposes a semantic analysis of possessive constructions based on an assumption that all posses- 
sives are base generated. Following Abney's (1987) DI hypothesis, Barker proposes the following syntactic analysis of possessives.

(5) John's mother

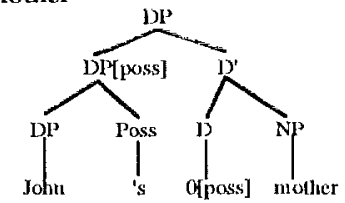

Ife claims that a relation from a possessor to a possessec is lexically determined if the possessee is a relational noun such as 'son', 'mother', etc. Such relations are represented by non-monadic predicates. For example, 'mother' will be translated by a dyadic predicate 'mother' such as follows:

\section{(6) $[$ [mother $]]=\lambda x \lambda y[$ mother $(x, y)]$}

When a possessee is not a relational noun, then the possessive denotes a relation based on gencral ownership or some sort of closeness relations, and Barker calls such relations an extrinsic relation. II pproposes the following two scmantic translations for possessive determiners corresponding to the above distinction.

$$
\begin{aligned}
& \text { a. } \left.\left[\left[0_{[\text {poss }]}\right]\right]=\lambda R \mid R\right] \\
& \text { b. }\left[\left[0_{[\text {poss }]}\right]=\lambda P \lambda x \lambda y[p(x, y) \& P(y)]\right.
\end{aligned}
$$

The translation in (7-a) is used in the casc of a lexical relation, while $p$ in (7-b) denotes an extrinsic relation such as ownership. When they are used in the analyses of "Jolnn's mother" and "Iohn's human", resulting translations are as follows:

$$
\begin{aligned}
& \text { a. [IJohn's mother }]=\lambda y[\text { mother }(j, y)] \\
& \text { b. }[\text { John's human] }]=\lambda y[p(j, y) \& \text { human(y)] }
\end{aligned}
$$

Note that 'mother' is a relational noun while 'human' is not. The above translations explain why the "human of John" is ungrammatical as opposed to the grammatical counterpart, "the mother of Joln". A noun, ' $Y$ ' in the form of " $X$ 's $Y$ ", can be either relational or non-relational, but ' $Y$ ' in the form of "the $Y$ of $X$ " must be relational. Therefore, a nonrelational noun such as 'human' cannot form an expression "the human of X". Barker's analysis provides a good foundation for our insight on relations in functional descriptions in gencral. Now, we say a nown $X$ is relational if and only if it allows both " $Y$ 's $X$ " and " $X$ of $Y$ ". All relational nouns are translated into non-monadic predicates.

In the above discussion, we did not inquire on the status of definite articles in the form of "the $Y$ of $X$ ". One may ask whether or not all relational nouns in the form of " $Y$ of $X$ " require definite articles, and the answer is obviously NO. It secms that only a certain set of relations possess the uniqueness presupposition on the arguments of the relations. For example, a relation 'mother-of $(X, Y)$ ' that means that $Y$ is the mother of $X$ possesses the uniqueness presuppesition on the sccond argument but not on the first argument. In contrast, a relation 'son-of(X,Y)' that means $Y$ is a son of $X$ has the uniqueness presupposition on the first argument but not on the second. Relations generally describe property/character- istic of one individual that occupies one argument of the relation. Let us call this argument the primary argument of a relation as oppose to the referential argument that links to a referent of the functional antecedent. In 'mother'-of $(X, Y)$ ' $Y$ is the primary argmment and in 'son-of $(X, Y)$ ' $Y$ is the primary argument. 'Thus, if a primary argument of a relation $Y$ is presupposed as unique, a detinite article is required in the form of "Y of $X$ ". This is the reason why 'mother' in 'mother of" requires a definite article but not 'son' in 'son of'.

Uniquencss presuppositions on certain arguments of relations are clcarly lexical in nature. Definite articles in IDI) reflect this lexically marked presupposition. Cases without uniquencss presupposition such as 'son-ol" should be called Functional Indefinite Descriptions (FID) (See Wada (forthcoming) for lurther discussion on this type of luuctional Anaphora).

\section{Accessibility}

Now, we extend the scope of our examination from possessive-based stiuctures to other cases such as 'a book' 'the duthor'. Since 'author' is a relational noun, we anticipate that the same kind of analysis is possible to the analysis of 'the author'. Irom the previous discussion, we know that the relational functional anaphoric link between 'a book' and "the author' is possible provided that "the author of the book" and "Ihc book's author" are both legitimate expressions. In other words, 'author' is a relational noun that denotes a dyadic lexical relation 'author'. 'The two arguments of the ielation are a referent for a salient book in a discourse and a referent that is the uniquely identifiable author of the book. However, the expression "the author" needs to be linked anaphoricaly to its lunctional antecedent, namely 'a book'. Kamp assumes that there is a selection set for this definite description and the most salient individual in the set will be selected as its antecedent. This is no different from regular definite description resolution. Nonetheless, we need to add some details to this.

As pointed out by Chicrchia (1993), the functional anaphoriclink must be constrained. Both Kamp and Chierchia assume that FDD can be analyzable as pronominal possessives. The anaphoric links that are interpreted from paraphrased pronominals and their antecedents must follow general constraints on pronominals. In DR'I, such a constraint is called the accessibility condition based on weak subordination relation ( $\geq$ ) between DRSs. Kamp and Reyle (1993:120) define it as follows:

\section{(9) Accessibility Condition}

Let $\mathrm{K}$ be a DRS, $\mathrm{x}$ a discourse referent and $\gamma$ a DRScondition. We say that $x$ is accessible from $\gamma$ in $K$ iff there are $K \geq K_{1}$ and $K_{1} \geq K_{2}$ such that $x$ belongs to $\mathrm{U}_{\mathrm{K} 1}$ and $\gamma$ belongs to Con $_{\mathrm{K} 2}$.

The above condition roughly tells that when an expression can be interpreted as anaphoric to a certain entity, that anaphoric expression must reside within some extention of 
the DRS in which the antecedent entity resides. FDD is no exception to this condition. Let us repeat the example of (1) and its variation here.

(10 a. Every book about Picasso made the author rich. b. Every book about Picasso was published by $\mathrm{Mr}$. King.

\#The author became rich.

The contrast shown in the above sentences is comparable to the following pair.

(11) a. Every book about Picasso made its author rich.

b. Every book about Picasso was published by

Mr.King.

\#Its author became rich.

The pronominal possessive, 'its', appears in exactly the same location in the above sentences as the FDDs in (10). It secms unarguable to assume that the two obey the same constraint. Indeed, it is more consistent to treat FDD as pronominal anaphora than to treat it as definite anaphora when we consider that referential arguments introduce regular discourse referents such as pronominals.

In sum, we observed that lexical relational FDD is licensed by lexical relations of the head nouns. The relations force uniqueness presupposition on the primary arguments of the relations. Furthermore, like pronominal anaphoric links, functional anaphoric links obey accessibility condition. In the following section, we examine other relational FDDs and see whether the above observed characteristics hold.

\subsection{FDD based on Situational Roles}

Certain relations do not keep regular sense of relations that would typically be held between two individuals. That is, those that we consider here usually do not allow paraphrasing "X's $Y$ " and "the $Y$ of $X$ " interchangeably. In this section, we consider a group of nouns that denote a relation between a situation and its unique element. Let us examine an example first.

(12) John attended a wedding last week.

The bride was his ex-girlfricnd.

Hawkins pointed out that both a speaker and a hearer must have a shared knowledge about a common situational setting. In (12), it is non-controversial to assume general knowledge that 'the bride' is a unique and necessary role in the situation of 'a wedding'. I claim that role nouns such as 'bride' are closely related to certain situations and that due to this close relationships, functional anaphoric links are possible. Nouns of typical social toles and professions such as 'president', 'referee', 'judge', 'lawyer', 'driver', 'victim', 'murderer' satisfy for this use of FDD (Lobner (1985:294)).

I assume a situational role noun is non-monadic predicate whose first argument (referential argument) holds a referent for a situation. For example, 'the bride' is represented in the following way.
(13)

\begin{tabular}{|c|}
\hline $\mathrm{x}, \mathrm{S}$ \\
\hline $\operatorname{bride}(\mathrm{S}, \mathrm{x})$ \\
\hline
\end{tabular}

' $S$ ' in (13) is a discourse referent for a situational role referential argument of "bride". Like functional antecedents of relational nouns, ' $S$ ' will be resolved with a functional antecedent. Some examples of situation setting nouns are 'wedding', 'court', 'case', 'incident', 'accident', 'class room', 'restaurant', etc. I call these words situation triggers. I assume that typical situation triggers and their FDDs must be available in the lexicon as part of common-sense knowledge of English. For example, 'bride' should mark its situation trigger 'wedding' in the lexicon. Of course, this is a trivial solution and we need to determine formal characterization of situation and situation triggers as well as more general solution based on common sense reasoning.

\section{Subordination and Situational Role FDD}

Because Situational Role FDD always appear with a certain situational setting, it is often the case that we see the following kind of contrast.

(14) a. Usually if John mects cvery pastor who administers a wedding, he writes to the bride.

b. \#Usually if John meets every boy who has an interesting book, he writes to the author.

Notice that since (14-a) and (14-b) are structurally identical, both 'wedding' and 'book' should not be accessible to 'bride' and 'author', respectively. $A$ s we see in (14), it is not the casc. Presumably, (14-a) is represented in the following DRS.

$\left(14^{\prime}-a\right)$

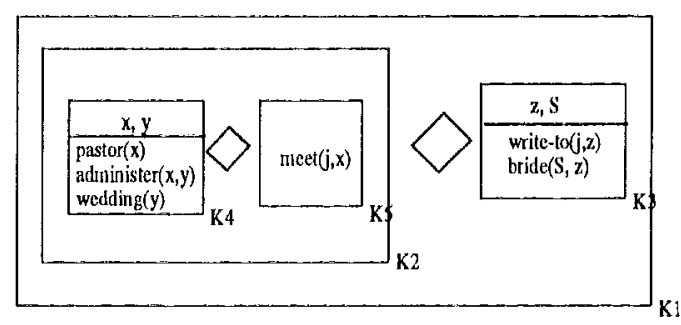

' $\mathrm{y}$ ' is in $\mathrm{UK}_{4}$ and is subordinated in $\mathrm{K}_{2}$. Therefore, it is clear that ' $y$ ' is not accessible to $S$ in $U_{K_{3}}$. The question here is why seemingly impossible link is allowed in (14-a) but not in (14-b).

It has been known that there are several cases in which the accessibility condition violation does not result in infelicitous anaphoric links. Roberts (1987) provides a DRT based analysis for a similar phenomenon with pronominal anaphora. Consider the following example.

(15) Harvey courts a girl at every convention.

She always comes to the banquet with him.

She claims that a pronominal 'she' is linked to 'a girl' since the second sentence is modally subordinated in the conse- 
quent of the DR conditional in the first sentence. In other words, the second sentence is under the quantification of the situation in the first sentence due to the fact that the modality appears in the second sentence. Notice that even in (15), an incidence of FDD is apparent: 'the banquet' is functionally linked to 'convention'.

In (14-a), we can safely assume that the consequent sentence is subordinate to the DR conditional's conscquent, while in (14-b) such explamation does not seem to be available. Once again, we see a certain similarity between pronominal anaphora and situational role FDD.

\subsection{FDD based on Temporal/Locational Kelations}

The third group to consider here consists of the following kinds of expressions.

(16) a. the moining of December 31 the spring of 1988

b. the midst of Ramadan the beginning of the wat

(17) a. the top of the house the edge of the bridge the side of the car the bottom of the bottle

b. the north of London

c. the middle of the bridge

Note that all of the head nouns in the above FDI) denote cither temporal as in (16) or locational points as in (17) with respect to other temporal or locative points. What makes these FDD distinctive from the two other relational FDDDs examined so far is 1) that they cannot be paraphrased by possessive construction of the form " $X$ 's $Y$ " although "the $Y$ of X" form is acceptable as shown above; that is, they are not lexical rclations, and 2) that they are not situational roles. liurthernore, as a group, these nouns typically link to functional antecedents that are auchored expressions.

Note that this observation allows us to consider certain close relationship between this subclass and expressions such as follows:

(18) the city of New York

the port of L_os Angeles

Fxpressions in (18) are usually considered as proper names, i.c., anchorcd expressions. Nonetheless, it is possible to consider 'the city of' as a function.

\subsection{DR'T Treatment of Relational FDD}

All of the relational HDDs ate tratnslated into nonmonadic relational predicates. The number of arguments depend upon relations lexically specificd in the lexicon for relational nouns. For example, most of kinship terms are dyadic predicates but some derived nominals will have the same number of arguments as the number of arguments that their verbal counterparts possess.

Nonetheless, at the time of translating a relational noun, whether or not the noun is used anaphorically, func- tional anaphorically, or non-anaphorically is not known. Therefore, we cannot select an appropriate DRS construction principle at the time of translation of FDD. What I would like to propose is that we translate FDD into non-monadic relation predicates but do nothing more than the translation at this time. I hypothesize that any un-instantiated referential argument introduces an anaphoric type reference marker. This reference inarker can be processed further in three ways: finding its antecelent, finding its functional antecedent, and finding neither its antecedent nor its functional antecedent. The following IDD construction rule states the above senario.

\section{(19) IDD CR}

Given a relational IidD phrase 'the N',

1) Introduce a relational condition, $R$, with an appropriate argument structure in $\mathrm{Con}_{\mathrm{K}}$.

2) Introduce a new reference marker, u, for a principle argument of $\mathrm{R}$ in $\mathrm{U}_{\mathrm{K}}$.

3) Introduce a set of new reference markers for the Iest of the arguments of $R$ in $U_{K}$.

4) Substitute $u$ for 'the $N$ ' in $p$.

Let us take an example of relational IDD and see how the above CR will be applied.

(20) Ysually if John buys an interesting book, he writes to the author.

At the time of translating the phrase, 'to the author', we have the following DRS under construction.

(21-1)

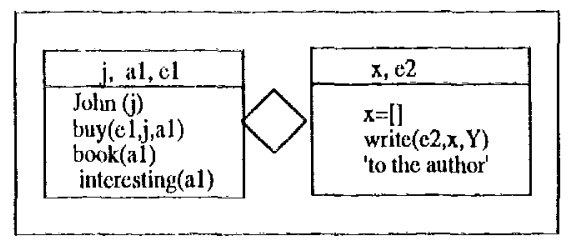

Since 'author' is a relational noun, the lexicon provides information concening its lexical denotation of the relation, namely a predicate "author' with two argument position. The above liDD construction rule produces a DRS as follows:

$(21-2)$

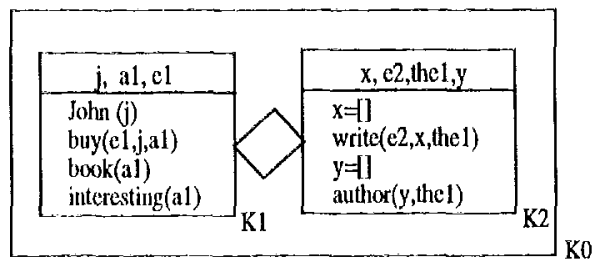

$\Lambda$ this moment, three things must be done: 1 ) resolve ' $x$ ', which is for the pronoun, 'he', 2) resolve 'thel', which stands for the entity of 'author', and 3) resolve ' $y$ ', that stands for the functional antecedent for 'author'.

Resolving ' $x$ ' with ' $j$ ' is trivial. ' $j$ ' is the only entity that is accessible to ' $x$ ' with gender, number satisfaction. How- 
ever, 'the1' cannot be resolved with any antecedent since there are no previously mentioned 'author' or deictically salient antecedent available from the above DRS. What we have to do is to accommodate it. Thus, we leave 'the1' in $\mathrm{U}_{\mathrm{K} 2}$ without doing anything. Now, we resolve ' $y$ ' with 'a1', an entity that stands for 'book'. 'al' is accessible to ' $y$ ' due to the extension of DRS $\mathrm{K}_{1}$ to $\mathrm{K}_{2}$. Note that this functional link is only possible when the lexicon provides common sense information that specifies the kind of relation that is held betwcen 'book' and 'author'. After these resolution operations, we have the following completed DRS.

(21-3)

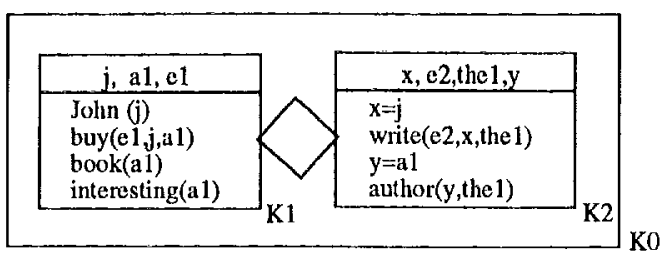

What happens when there is a previously mentioned phrase? Consider the following example.

(22) Usually if John meets the author of a book, he praises the author.

The first incident of 'author' is the case of explicit FDD in Kamp (1984). Its functional antecedent is provided by 'of' phrase. The second occurrence of 'author' is non-functionally linked to the initial mention of 'author'. I assume that the second incidence of 'author' introduces the same relational condition. And, due to the non-functional link to the initial incidence of 'author', the arguments will be filled with the exact copies of the arguments of the initial 'author'. 'Thereforc, we have the following DRS.

(23)

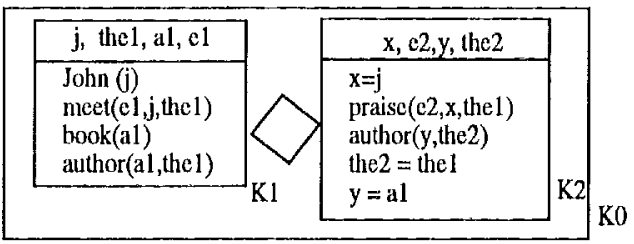

Note that when ' $y$ ' is linked to 'a1' via a regular anaphoric link of 'the2' to 'the1', it is not necessary to invoke another anaphora resolution procedure for ' $y$ '. The situation is just the same as in the case of explicit FDD such as 'the mother of John'. The first argument of a relation 'mother( $\Lambda$,the1)' is syntactically connected to entity denoted by the 'of' phrase.

\section{NON-RELATIONAL FDD}

$\triangle$ group of FDD that we call non-relational FDD are FDDs such as "the tallest man", "the third book", and "the same girl". They all take obligatory definite articles but are first mentions without having regular sense of antecedents. However, the references of the three examples are determined with respect to some sets of individuals in the dis- course. This certainly satisfies our definition of FDD. Nonetheless, the function and the mechanism underlying the function differ substantively from relational FDD discussed so far. In this section, we will see how they differ and I will sketch a treatment for this class of FDD briefly. I would like to refer readers to the work (Wada (forthcoming)) for further and detailed discussion on non-relational FDD.

First of all, relationalFDD and non-relation FDD differ structurally. A typical syntactic structure of relational FDD is an NP of the form $\left[_{n p}\left[_{\text {det }^{\text {the }}}\right],\left[_{n b a r}\left[_{n} A\right]\right]\right] . N$ (lexically ' $A$ ' in the form) must be a relational noun and it can take a complement 'of' phrase. Non-relational FDD, on the other hand, must contain an adjunct phrase headed by one of elements such as superlative adjectives, ordinal numerals, and identifying adjectives like 'very' and 'same'. Thus, its typical form is $\left[_{n p}\left[\left[_{\text {det }}\right.\right.\right.$ the $],\left[_{n b a r}\left[x, ' A\right.\right.$ '], $\left[_{n}\right.$ ' $B$ ']]]. ' $A$ ' is the FDD licensing lexical element and ' $B$ ' is any noun.

Second, the functional link of non-relational FDD is licensed by a head of the adjunct phrase such as a superlative adjective, an ordinal number, or an identifying adjective rather than a head noun (relational noun) of FDD. I call these heads of adjunct phrases functional modifiers. Functional modifiers denote functions that introduce discourse referents which will be resolved with functional antecedents in a discourse.

Third, non-relational FDD takes a selection set instead of an object as their functional antecedents. $A$ selection set is a maximal set of objects in a given discourse that satisfies descriptions in the head nouns of the non-relational FDD. The phenomenon is reminiscent to plural anaphora where the antecedents are usually maximal sets.

Finally, the uniqueness presupposition of the referent of non-relational FDD is provided by the lexical/semantic characteristics of the functional modifiers while it is due to the lexical relation of the head noun in relational FDD.

In this paper, we examine three subclasses of this FDD: superlatives such as 'the strongest man', ordinal number modification such as 'the third book', and identifying adjective modification such as 'the same car'.

\section{Basic Analysis of Non-relational FDD}

Kamp (1984) provides an analysis of superlative constructions. In that, he treats superlatives as a set of comparalives under a universal quantifier that introduces a DRT-conditional. The set of comparatives are distributed over members of the selection set given in the discourse. Kamp shows this selection set as analogous to 'among them'. Consider the following.

(24) Three men came to harvest rice.

The weakest man operated a combine machine.

The superlative 'the weakest man' takes maximal set of 'the three men who came to harvest rice' as its functional antecedent and returns the unique individual that satisfies a condition that this individual is weaker than any member of the set except himself. The following DRS represents Kamp's 
treatment of superlatives.

(24')

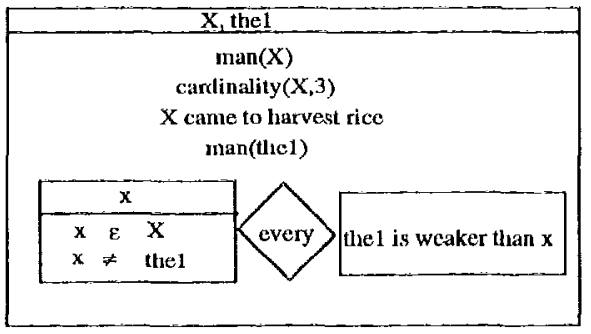

In our treatment, we add a dyadic predicate 'weakest (X,the1)' to the above representation to indicate that the the set of three men is the functional antecedent of this FDD and the set is distributed over members of the set.

Both ordinal numeral phrases and identifying adjectives are analyzed to have the same logical structures as the superlative adjectives discussed above. The ordinal numeral case is based on enumeration operations via DRT-conditional instead of a set of comparisons under an universal quantifier. Once enumeration operation takcs place, each of the members of a set can be referred with an index, the ordinal number. The identifying adjective case is treated exactly like the case of superlatives.

\section{IMPLEMENTATION OF FDD RESOLUTION}

ITP's Interpretext natural language understanding system has been under development in the past few years. We reported some early results in MUC3 Conference and elsewhere (Dahlgren, et al. 1991). The system includes a large Naive Semantic lexicon, a principle-based wide coverage parser with a sense disambiguation mechanism, a DRS construction module, an anaphora resolver, and lexical and discourse database handlers. FIDD resolution was implemented as part of a large anaphora resolution mechanism.

In the implementation, scarching order among hypotheses is very important. Certain ordering eliminates possibilities of available resolution, and other cases cause increase in processing load of the resolution. Thus, our goal is to reduce the processing load as much as possible by reducing search space and to reduce resolution crrors by setting item specilic hypotheses ordering at the same time.

All of the [PDD are potential instances of simple subsequent-mention DD. For example, a DJ with a lexical relational noun such as 'the mother' may have a regular antecedent such as 'a mother' in the discourse. Although first-mention DDs that include FDDs are statistically more common than typical subsequent-mention IDDs, a possibility of being subsequent-mention DD should be tested first. This is because of the fact that the range of description satisfying antecedents are more constrained than the range of functionally satisfying antecedents.

Generally, we should hypothesize more restricted assumption before general ones. In our implementation, each FDJ subclass possesses its own set of ordered hypotheses. For example, temporal/locational relation FDD typically have anchored expressions as their functional antecedents and our resolution module searches in this restricted area (an anchored object list) before it searches in the previous discourse. For another example, situational role FDD checks simple subsequent-mention case first; if it fails, then it tries to find a situation-trigger in the accessible universc of discourse. Currently, we have been conducting a large scale evaluation on anaphora resolution.

\section{CONCLUSION}

This paper examined Functional Definite Descriptions and proposed two types of IDDD, relational and non-relational. The analysis presented here was based on claims that FDD introduce discourse referents of pronominal type, and that functional anaphoric links obey the same accessible conditions that pronominal anaphoric links obey. FDD is closely related to functional anaphoric expressions in other categories such as Functional Indefinite Descriptions. To understand FDD better, more research on functional anaphoric expressions in general will be needed in the future.

\section{REFERENCES}

Asher, N. (1993) Reference to Abstract Objects in Discourse, Kluwer Academic Publishers.

Barker, C. (1991) Possessive Descriptions, Ph.D. thesis, University of California Santa Cruz.

Chierchia, G. (1993) Anaphora and Dynamic Interpretation, LSA Summer Institute Course material.

Cooper, R. (1979) "The Interpretation of Pronouns", in Syntax and Semantics, Vol. 10, eds. F. Heny and II. Schnclle, Academic Press: New York.

Dahlgren, K. et al. (1991) "ITP: Description of the Interpretext System as used for MUC-3," in Proceedings of Third Message Understanding Conference, pp. 79-83, Morgan Kaufman Publishers.

Engdahl, E. (1986) Constituent Questions, Reidel: Dordrecht.

Fraurud, K. (1990) "Definiteness and the Processing of Noun Phrases in Natural Discourse" in Journal of Semantics, Vol.7:pp.395-433.

Hawkins, J. (1978) Definiteness and Indefiniteness, Croom Helm: London.

Kamp, II. (1984) "SID Without 'Time and Questions", manuscript.

Kamp, H. and U. Reyle (1993) From Discourse to Logic, Kluwer Academic Publishers.

Lobner, S. (1985) "Definiteness", in Journal of Semantics, Vol. 4: pp.279-320.

Roberts, C. (1987) Modal Subordination, Anaphora, and Distributivity, Ph.D. thesis, University of Massachusetts.

Wada, H. (forthcoming) "Functional Anaphoric Expressions", manuscript. 